\title{
Research on Evacuation Simulation of Large-scale Stadium Staff
}

\author{
Juhai Wang \\ Institute of Physical Culture, Weinan Normal University, Weinan, Shaanxi, 714099
}

Keywords: large-scale gymnasium; evacuation simulation; simulation research; personnel safety

\begin{abstract}
Large-scale stadiums are crowded and extremely important. Once emergencies occur, they can cause major economic losses and sometimes cause serious casualties. In recent years, performance-based fire protection designs have been continuously optimized, and computer simulation studies have been used as the main means of performance-based evacuation design. However, at present, the number of gymnasiums has increased, and the problem of safe evacuation has become a center of concern for all walks of life. Therefore, this paper analyzes the characteristics of evacuation of large stadiums, and then conducts in-depth research on existing problems in evacuation simulation simulations and proposes corresponding modification suggestions. It is expected to provide personnel with more accurate design when building evacuation models, to make up for previous deficiencies, to help designers better evaluate the safety and reliability of building fire protection design, and to provide reference for ensuring the safety of large-scale stadium staff.
\end{abstract}

\section{Introduction}

With the rapid development of China's economy, large-scale gymnasium construction has continued to increase, such as the landmark "Bird's Nest" and "Water Cube". In addition to the capital, there are also many stadiums established in Shanghai, Guangzhou and other places, such as the World Expo site in Shanghai, the International Gymnastics Center badminton hall. Guangzhou's Olympic Sports Center, swimming diving hall and other large-scale sports buildings are all landmark buildings in China's sports venues. As a gathering place for daily life, gymnasiums are diverse and integrated, and their overall utilization rate is constantly increasing. The gymnasium can not only be used as a stadium for sports events, but also can be used as venues for theatrical performances, political gatherings, and so on. This requires that the gymnasium be flexible and change the spatial combination to improve the comprehensive use value. However, this commercial construction has brought serious fire hazards and stampede accidents to the gymnasium, causing serious negative impact on the society. Therefore, the evacuation design of the gymnasium and the reduction of accidents have become the focus of attention of the society.

\section{Overview of Accident Hazards in Large-scale Stadiums}

The number of modern gymnasium buildings is increasing, and it is used as a sports competition venue only. Its utilization rate is relatively low, and the daily maintenance expenses are relatively large, thus making the finance useless. Therefore, in order to increase its utilization rate, large gymnasium buildings will be transformed into public gathering places for daily life, making them more diversified and commercialized, and improving the overall utilization rate of gymnasiums. In addition to holding some important sports events, the stadium now holds art performances and large-scale political gatherings. This requires that gymnasiums be flexible and changeable. Through space combinations, mobile facilities can be adjusted to achieve relevant functions and improve the efficiency of comprehensive utilization. During the transformation of the stadium building, there will be many potential safety hazards. For example, in the Huanglong Stadium in 2010, in the process of holding a concert, the fire caused by the charge exceeded expectations.

As a commercial large-scale public building, a large stadium is also an important place for the public to gather. The accumulation of large numbers of people leads to the occurrence of safety 
accidents. There are two major security risks, namely stampede events and fire incidents. A fire or stampede in a gymnasium can cause casualties and economic losses, as well as serious negative impact on society. There are many reasons for the fire, such as illegal operations. The 2008 Olympic Sports Center Stadium was caused by this reason. This is because during the construction of the Olympic Sports Center Gymnasium, the construction of fire fighting facilities was not emphasized, which led to the inability to control the fire at the initial stage, the fire lane was occupied, etc., all of which led to the fire. In addition, human factors are also the main cause of safety accident products. In the event of a fire, people will panic, the crowd will be disorderly, the export will be blocked, and the stampede will eventually occur. Large gymnasiums are an important place where crowds gather. Therefore, it is necessary to do a good job of evacuation simulation and simulation, and it is also an important issue for China's current research.

\section{Status Quo of Simulated Evacuation Simulation in Large-scale Stadiums}

The evacuation simulation research of large-scale gymnasiums in China started in the period of the "Tenth Five-year Plan". However, only a few experts conducted research on it, and there are even fewer experts who specialize in evacuation simulation and research for large stadiums. Then in 2008-2110, the success of many sports games was held, and all walks of life paid enough attention to the evacuation simulation and research of large stadiums. At that time, there was no special fire code in China. There were only two fire codes with the county officials of the gymnasium. The Code for Fire Protection of Architectural Design and the Code for Design of Sports Buildings did not contain detailed evacuation regulations for the gymnasium. There are many problems with the evacuation design. It should be noted here that the "Code for the Design of Sports Architecture" is not yet a professional manual for fire protection technical specifications. It does not have the corresponding authority and is not recognized by the society.

The 2008 Olympic Games stadium fire safety design index mainly refers to the British publication "Guides to safety at sports Grounds", which is a more authoritative stadium evacuation design specifications, the main purpose is to guide the safety design of the stadium building. However, for our country, it does not apply, because the construction conditions of large-scale gymnasiums in our country exceed the safety norms of books and can only be optimized. At present, China has professionally sorted out and analyzed the performance of the gymnasium according to its functional characteristics to complete the evacuation design of the gymnasium. Performance optimization can be based on the characteristics of the building, the existence of hidden dangers, such as fire hazards, personnel evacuation situation analysis and evaluation, and then use the computer to simulate the fire evacuation simulation, and ultimately find the corresponding solution. This design has been widely used in the construction of large stadiums in China, such as the Water Cube and the Bird's Nest. Computer simulation is the key to performance design. The research of evacuation simulation originated in the mid-1980s. Through the simulation of the evacuation of the interior of the building, it can be intuitively understood whether the evacuation design of the gymnasium has reached the expectation.

\section{The Evacuation Method Commonly Used in Gymnasiums}

The method of evacuation of the masses in the stadium is divided according to the departure route. There are several types, such as up/down, middle, side, and composite. Up/down evacuation and side-type evacuation are usually used in small and medium-sized gymnasiums. Up/down evacuation methods are used to effectively save auditorium area and evacuation time, and increase the use rate of the gym hall, but it is prone to confusion. , resulting in stampede accidents; and the use of side-type evacuation methods, can ensure the seat space integrity, economical, but limited, and poor versatility.

Large stadiums often use intermediate evacuation and compound evacuation. There are two types of intermediate evacuation: evacuation without transverse channels and lateral evacuation. It integrates the advantages of both up and down modes, diverts personnel, improves the efficiency of 
space utilization under the stands, and ensures the smooth flow of people to clear the channels. However, it will occupy most of the seating space with a good view, and it will have poor economic efficiency. The compound evacuation method is the integration of commonly used evacuation methods. It is mainly applied to the circular/elliptical plane gymnasium and has a unique shape. The compound evacuation method is rich in many talents and unique in shape. It can not effectively improve evacuation efficiency, save seating space, and is economical and flexible. However, it has high requirements for the ability of designers and the flexibility of evacuation methods, and the evacuation method is complex. It is also difficult for temporary migrants to find suitable outlets.

\section{Research on Evacuation Characteristics of Large-scale Stadium Gymnasts}

The gymnasium building is organized for the purpose of hosting sporting events. Therefore, a large area is required for fixed seats. This makes it difficult for the stadium to clearly define the fire zone in accordance with regulations. The span of the gymnasium is larger, and the required building materials are more demanding. The most deadly thing is that the gymnasium building is poor in fire resistance and easily collapses when it encounters high temperatures. Secondly, there are many styles of competition halls. In order to adapt to different external conditions and meet various competition demands, it is difficult for people to increase evacuation.

The width and number of internal steps in the stadium are all calculated, and the width of the steps is greater than the capacity of the evacuation exit, and the step height of the steps is proportional to the slope of the stands. The higher the level, the more dangerous the evacuation. In addition, the width of the channel steps is inconsistent, which can affect the evacuation speed of the personnel. The broadband of the steps is inconsistent, which reduces the walking speed of the personnel, and thus reduces the evacuation efficiency. Therefore, the width of the tread of the stadium building stairs should be consistent, but these are difficult for the stadium building. For example, in Fudan's stadium in Shanghai, the seat spacing is $820 \mathrm{~mm}$, and the difference is $450 \mathrm{~mm}$. In order to maintain the level with the seat, the width of the steps is $250 \mathrm{~mm}, 250 \mathrm{~mm}, 320 \mathrm{~mm}$, and the width of each level cannot be guaranteed.

\section{Research on Evacuation Simulation of Large-scale Stadiums}

The gymnasium stands in a special space and has a high flow of people. Therefore, it has become the focus of evacuation design. The evacuation area includes the three main parts of the stands, walkways, and exits. When evacuating, they leave the seats, walk through the aisles to the longitudinal passages, and then pass through the corridors and doorways to a process of reaching the safe area. Staff in the seating area are dense and activities are limited. Therefore, when designing, the design must be carried out strictly in accordance with the relevant regulations. For example, each room in the room should not exceed 26 rows and outdoors should not exceed 40; if the longitudinal walkway has only one side, The number of seats should be halved.

At present, China uses three types of evacuation simulation software, namely Simulex, Pathfinder, and Building Exodus, and then use them to model seating areas and stair seats. However, Simulex and Pahtfinder do not have special modules to simulate the seat area. When moulds are used, they can only be separated by ordinary means, that is, the use of guns instead of seats restricts the personnel, which results in errors in the simulation data. In the simulation of the evacuation of the step-type seat, the simulation can only be performed in the plane-type seating area, which will still cause errors. Therefore, to ensure the accuracy of the data, the width of the boundary layer must be estimated. When the evacuation simulation is performed, the average evacuation speed of personnel can be reduced, and the width of the boundary layer can be judged.

Ladder-type vertical channel evacuation is the focus of evacuation simulation research in large stadiums. Simulex and Pathfinder are often used for modeling optimization. Simulex's modeling process has three steps. In the first step, each row of seats in the seating area is separated by lines, and the direction of movement of the personnel is controlled to ensure that the personnel and the seat move in the same direction; the second step is the seats in each row. The outlet is set with a link 
that connects the stairs to each other, and a corresponding link is also set on the stairs corresponding to the stairs. The third step is to add the seat area personnel and set the final exit.

When using the Pathfinder software to simulate the evacuation simulation of a gymnasium, it is first necessary to model the stadium's architecture such as rooms, floors, and exits. It should be noted here that pathfinder cannot model ladder seats and generally treats them as planes, which creates errors. However, Pathfinder can process ladder-type steps, so in the process of simulation, the stairs are modified to achieve the goal of evacuation. The common modeling approach is to constrain the establishment of a non-planar seating area in the seating area, then calculate the height of each level, create a room on the same height plane, and set the corresponding width parameters, outlet parameters, etc. To restrict the direction of movement of personnel to ensure that the direction of movement of personnel is consistent with the direction of movement of agents. Finally, at each level of the longitudinal passages, the upper and lower rows of seating areas are connected by small stairs to complete the approximate modeling of the seating area.

\section{Conclusion}

The gymnasium is a public building with a large population density. Its own structure is also very complex. It has a large space and a comprehensive nature. It makes the evacuation of the gymnasium staff more difficult, causing fire and stampede. Therefore, this paper analyzes the structural features and commonly used evacuation methods of the gymnasium, combines the relevant domestic and foreign safety norms, and uses the simulation software to model and deal with the evacuation of large gymnasium personnel. According to the evacuation simulation, the modeling process is optimized to improve the evacuation efficiency of large-scale stadium staff and ensure the safety of personnel.

\section{Acknowledgements}

This work was financially supported by Basic Research Projects of Weinan Technology Bureau(2017JCYJ-2-5) and Shaanxi Social science 2018 projects of major theoretical and practical problems research(2018Z001).

\section{References}

[1] Huo Ran, Yuan Hongyong Performance Analysis and Design of Performance Building Fire Protection [M] Hefei: Anhui Science and Technology Press 2003

[2] Xie Dayong, Tang Hai, Li Tao. Application of personnel evacuation analysis in the design of stadiums [J] Fire Science and Technology 2005, (03)

[3] An Qingxin Example and Analysis: Performance-based Safety Evacuation Design of Modern Stadiums [J] Huazhong Architecture 2006, (07)

[4] Zhang Wei Research on evacuation design in stadium building design [D] Tongji University 2007, (02)

[5] Beijing Olympics Project - Performance Fire Protection Design and Fire Safety Management [M] Beijing: China Building Industry Press 2009 